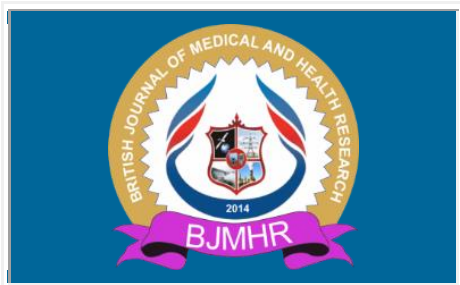

\title{
BJMHR
}

British Journal of Medical and Health Research

Journal home page: www.bjmhr.com

\section{Mosaicplasty for Osteochondritis Dissecans of the Knee: About 4 cases}

\section{Yassine Rachdi*, Adnane Lachkar, Abdeljaouad Najib, Hicham Yacoubi. Department of traumatology and orthopedics, Mohammed VI university hospital center, Oujda, Morocco.}

\section{ABSTRACT}

Osteochondritis dissecans (OCD) of the femoral condyles is a subchondral bone necrosis, resulting in osteochondral fragmentation that causes joint dysfunction. Mosaic grafting consists of harvesting osteochondral grafts from a donor site and transferring them to the osteochondral defect. The objective of our study was to evaluate the results of mosaicplasty as a treatment for osteochondritis dissecans of the knee. This is a retrospective study of 04 cases of osteochondritis dissecans of the knee in the department of traumatology and orthopedics at the University Hospital Oujda, Morocco. The average preoperative IKDC functional score was 43. The lesions were classified according to Cahill's classification in zone 2 and 3, and according to Harding's classification in zone B and C. According to Bedeouelle's classification, the evolutionary stage was divided into one stage IIb case, two stages III, and one stage IV.MRI revealed two stage II cases and one case for each of the stages IV and V according to Hefti's classification. Our surgical treatment consisted of osteochondral reconstruction of the injured area by mosaicplasty. A first arthroscopic step allows us to evaluate the size and depth of the cartilage defect. The 4 patients had an OCD grade 4 ICRS. All our mosaicplasties were performed open after a medial arthrotomy. Progressive rehabilitation was started the next day, and weight-bearing was not allowed until the sixth week. The IKDC score was 84.5. The arthroscopic "second look" showed the integration of the grafts with the edges of the healthy hyaline cartilage, creating a "golf ball" appearance. The mosaic graft is a validated cartilage restoration technique ${ }^{14}$. Garretson et al 15 demonstrated that the optimal and least constrained site was the margins of the superomedial trochlea. Hangody et al ${ }^{18}$ reported in a heterogeneous series of more than 1,000 mosaic grafts: $3 \%$ morbidity, four infections and 36 hemarthroses. Gudas et al ${ }^{22}$ compared 29 mosaic grafts and 29 microfractures for lesions with an average size of $2.7 \mathrm{~cm}^{2}$, and found a remarkable advantage of the mosaic graft in this indication. Mosaic grafting has the advantage of being less expensive than reconstructive techniques, performed in a single surgical step, and of offering immediate restoration of the cartilage surface.

Keywords: Osteochondritis, knee, mosaicplasty, grafting. 


\section{INTRODUCTION}

Osteochondritis dissecans (OCD) of the femoral condyles is a more or less extensive subchondral bone necrosis that can affect different parts of the knee ${ }^{1}$. The result is osteochondral fragmentation leading to joint dysfunction.

The aim of the therapeutic measures is to fill in the loss of substance in order to restore joint congruence and, if possible, to induce hyaline healing and thus to avoid arthrosic degeneration in the medium and long term. They can be classified as repair by subchondral stimulation, which most often leads to fibrous scarring (microfractures, Pridie perforations), reconstruction by bringing mature cartilage into the osteochondral unit (mosaicplasty and massive allograft), and regeneration by autologous chondrocyte culture grafting with the aim of achieving hyaline repair ${ }^{1,2}$.

Mosaic grafting, a repair technique, consists of harvesting osteochondral grafts from a donor site and transferring them to the osteochondral defect. This technique was Developed by L. Hangody in Hungary in the early 1990s ${ }^{3}$, a first symposium of the French Arthroscopy Society was devoted to this technique in 1999 and showed satisfactory short-term results, which have been confirmed by several authors ${ }^{4}$. Nevertheless, the longer-term results remain underestimated with small numbers and a very heterogeneous literature ${ }^{5}$. The objective of our study was to evaluate the results of mosaicoplasty as a treatment for osteochondritis dissecans of the knee in the short and medium term.

\section{MATERIALS AND METHOD}

This is a retrospective study about 04 cases with osteochondritis dissecans of the knee treated by mosaicplasty in the department of traumatology orthopedics CHU Oujda Morocco, spread over a period of 3 years between the years 2015 - 2018.

The exploration of the lesion included simple radiographs, classified according to the classification of Cahill and Berg of Harding, and Bedeouelle, these three classifications allow respectively the measurement of the length of the lesion, its width and give us a semiological appreciation of OCD MRI with its two sequences FSE T2 and 3D GRE T1 allows the lesion to be classified according to Hefti's classification The results were analyzed according to clinical (IKDC score), radiological (MRI classification of Mocart) and arthroscopic criteria by a "second look".

\section{RESULTS AND DISCUSSION}

All our patients were male with a mean age of 27.7. Pain was the main reason for consultation for our patients, there was only one case of joint impingement, joint mobility was preserved in all four cases, and a varus deformity of two finger crooks was noted in only one patient. The average preoperative IKDC functional score was 43. All of our patients had 
a standard radiograph of the knee, front and back. The lesions were classified according to Cahill's classification (frontal plane) in zones 2 and 3, and according to Harding's classification (sagittal plane) in zones B and C. According to Bedeouelle's classification, the evolutionary stage was divided into one stage IIb case, two stages III, and one stage IV (Figure 1).MRI revealed two stage II cases and one case for each of the stages IV and V according to the Hefti classification (Figure 2).

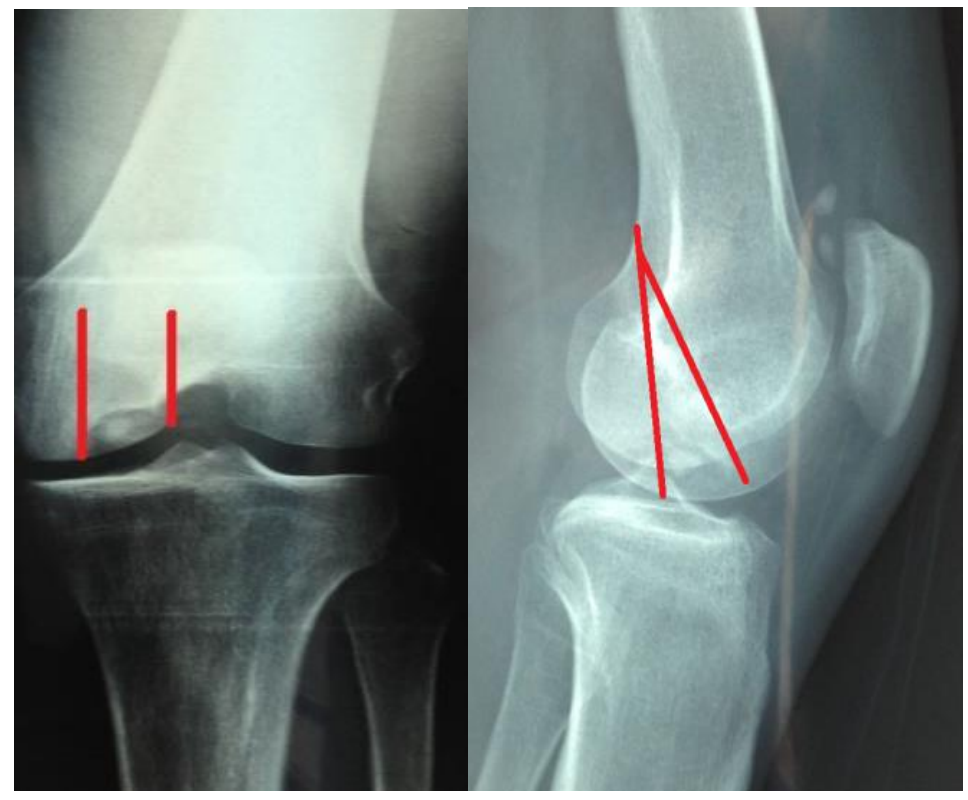

Figure 1: X-ray showing OCD stage IIb according to the Badeouelle classification.

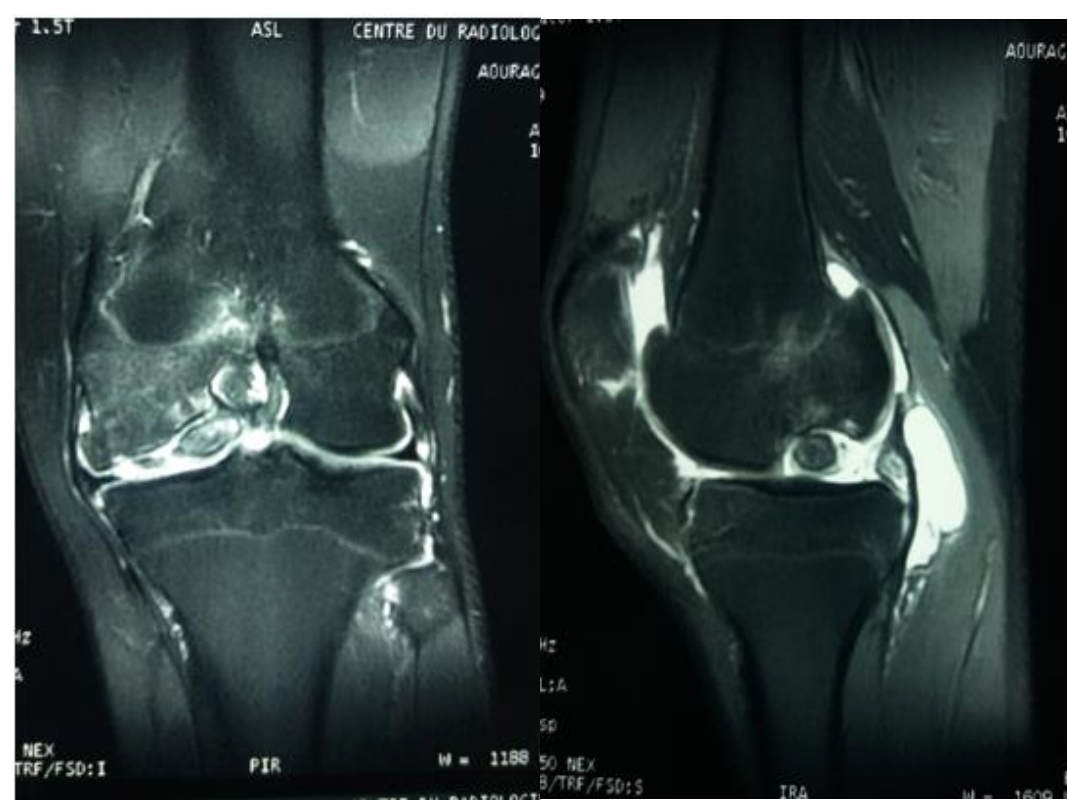

Figure 2: MRI of the knee showing OCD stage IV according to the Hefti classification.

We opted for a surgical treatment for all our patients. It consists of an osteochondral reconstruction of the injured area 'at the level of the medial condyle for our study' by mosaicplasty. A first arthroscopic step allows a complete joint assessment to be carried out, and to debride the edges and the bottom of the lesion in order to assess the size and depth of the cartilage defect, this assessment is objectified by the ICRS classification, the 4 patients 
had an OCD grade 4 ICRS. This preliminary assessment of the dimensions also allows the number of grafts required to be determined and the appropriate diameter to be chosen. No meniscal or ligament injuries were noted, nor were there any chondral injuries in the other compartments. All of our mosaicplasties were performed open after a medial arthrotomy, as the defect area exceeded $2 \mathrm{~cm}$ in all cases. We started drilling the receptacle with the graduated drill bit corresponding to the required diameter. The depth of drilling was $25 \mathrm{~mm}$. The graft was then harvested from the superior-medial area of the trochlea margin using a specific trephine. The grafts were inserted one by one using a graduated mandrel, in order to control the progress and adjust the height of insertion of the grafts (Figure 3).

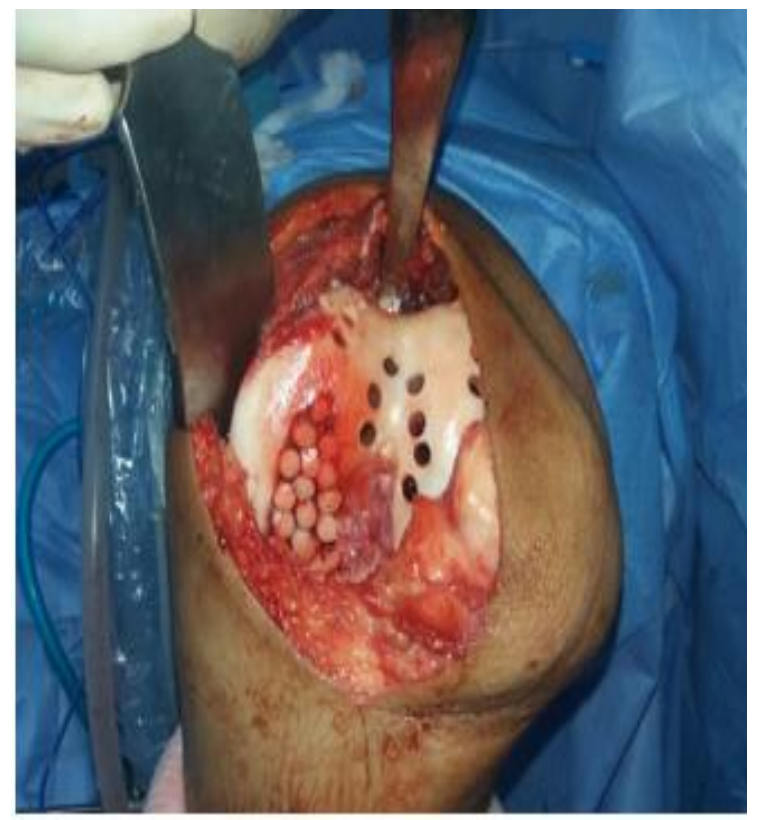

Figure 3: intraoperative image showing the sites of the removal and grafting of the osteocartilaginous plots.

The knee is immobilized by a rigid orthosis, cryotherapy and static work of the quadriceps is started the next day. Active mobilization of the knee was started at 21 days and weightbearing was not allowed until the sixth week. The IKDC score was 84.5 with a follow-up of 27 months. All our patients had a second look control arthroscopy which showed the integration of the grafts with the edges of the healthy hyaline cartilage creating a "golf ball" aspect (Figure 4). Unfortunately, in our patients, only one control MRI was carried out which showed a filling of more than 80 percent with good integration of the grafts (Figure 5), and a restitution of the subchondral lamina as well as the absence of necrosis. 


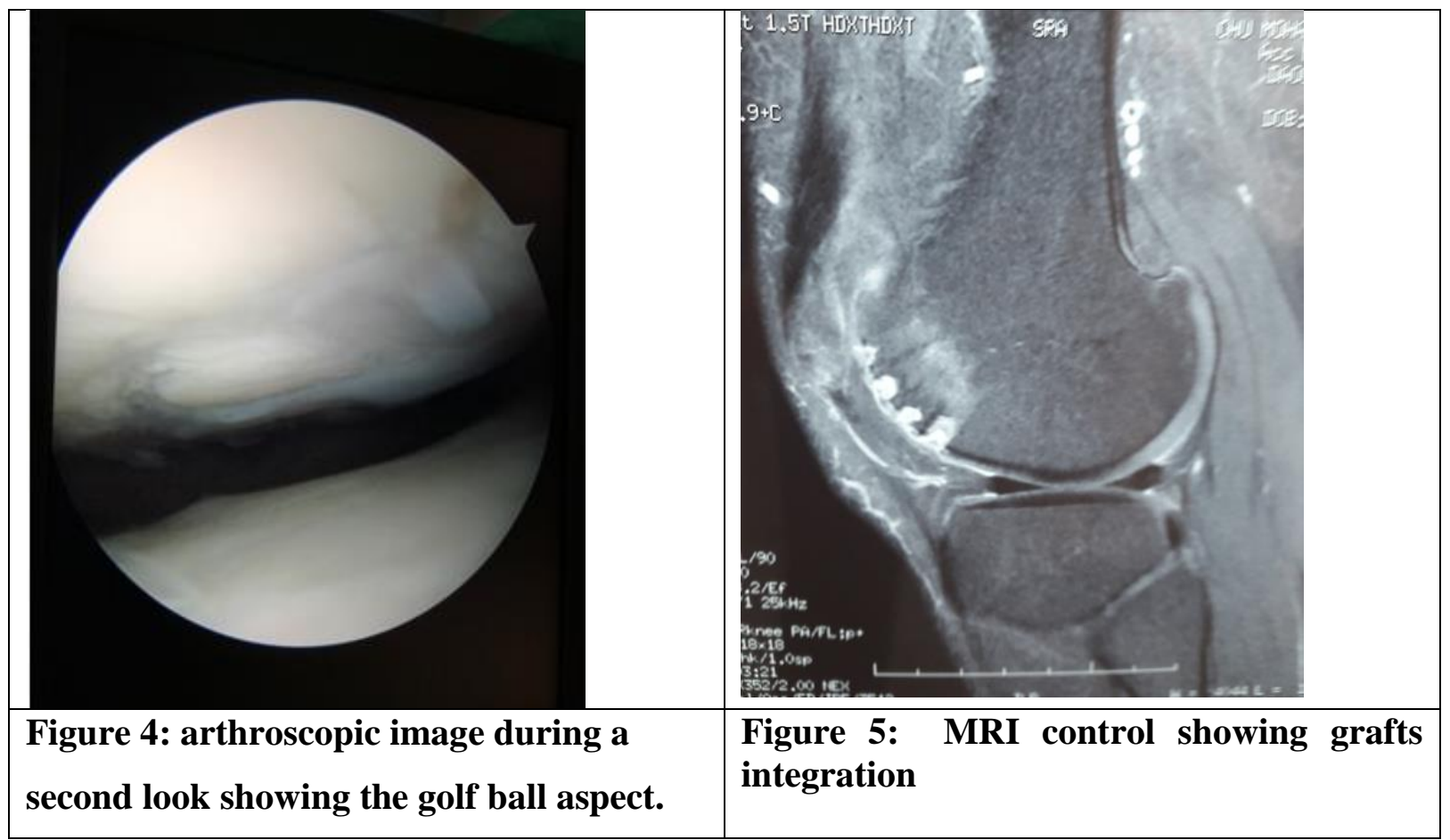

DISCUSSION:

Most authors ${ }^{6,7}$ report a male predominance. This could be explained by the fact that adolescent males have a greater propensity for sports than females, and therefore greater stress on their joints. Clinically, pain is the main symptom, as in our series. There are other less constant clinical signs with, in decreasing order of frequency: locking, hydarthrosis, catching and a decrease in joint mobility ${ }^{8}$. Standard radiography (face, profile and incidence of the notch for the more posterior forms) remains the first-line examination for the diagnosis and follow-up of the lesion ${ }^{8-10}$. It is essential and serves as a basis for any further investigations ${ }^{8}$. The three-quarter view is reserved for early and small volume forms ${ }^{8}$. MRI is the reference examination in terms of performance and safety ${ }^{8,11-13}$. Arthro-MRI takes longer and is more invasive ${ }^{8}$. Arthroscanner is not recommended because of its iatrogenic risk. Arthroscopy is the last resort since it allows visual inspection of the lesions and the use of a palpator, but it should not be used systematically to explore the lesions.

Mosaic grafting is a validated cartilage restoration technique ${ }^{14}$. Various studies have focused on the harvesting site. In 2005, Garretson et al ${ }^{15}$ demonstrated that the optimal and least constrained site was the edges of the superomedial trochlea. The morbidity of this harvest is not zero. It has been estimated 0 to $36 \%$ in the literature ${ }^{16-18}$. Reddy et al ${ }^{16}$ reported that, following harvesting of healthy knees for Talus lesions, four out of 11 patients had a poor outcome at four years' follow-up. Iwasaki et al ${ }^{17}$, on the other hand, reported no complications following harvesting of humeral lesions at two years' follow-up. . As regards the size and number of blocks, Robert ${ }^{19}$ reported, in his teaching lecture, the respective advantages of small and large blocks. plots. Thus, the larger blocks offer greater stability, less fibrous interposition, and a larger cartilage surface area, which is more important for the 
cartilage surface, at the cost of more difficult filling in the case of multiple blocks and a probable greater harvest morbidity. Sgaglione et al ${ }^{20}$, in his AAOS teaching lectures, recommended 6 to $8 \mathrm{~mm}$ wide stents with a length of between 15 and $20 \mathrm{~mm}$. On the other hand, this technique is not without complications. Hangody et al ${ }^{18}$ reported in a heterogeneous retrospective series of more than 1000 mosaic grafts: $3 \%$ morbidity, four infections and 36 hemarthroses. This rate was 13\% in the series presented, with a preponderance of hemarthrosis. In the same article, Hangody et al ${ }^{18}$ also evaluated the role of lesion location. They found a positive influence of medial condyle lesions compared to lateral condyle and femoropatellar lesions, with $92 \%$ of good and very good results for condyle lesions.

Regarding MRI evaluation, Tetta et al ${ }^{21}$ studied 24 patients treated with mosaicoplasty at nine years' follow-up, with $75 \%$ complete integration of the graft, and a correlation between the Mocart score and the clinical results was observed.

In comparison between the other techniques, the palliative methods have the advantage of a lesser blow and the absence of harvesting. Gudas et al ${ }^{22}$ compared 29 mosaic grafts and 29 microfractures in a prospective randomized study for lesions with a mean size of $2.7 \mathrm{~cm} 2$ at 3 years follow-up. The authors reported a remarkable advantage of mosaic grafts in this indication. Indeed, the mosaic graft group obtained $93 \%$ of good or very good results compared to $49 \%$ for microfractures. Horas et al ${ }^{23}$ compared mosaic grafting and firstgeneration autologous chondrocyte grafts associated with a periosteal flap, for lesions of 3.7 $\mathrm{cm} 2$ on average. No significant difference could be demonstrated.

\section{CONCLUSION}

Mosaic grafting thus appears to be a reliable technique in the short and longer term. It has the advantage of being less expensive than reconstructive techniques, performed in a single surgical step, and of offering immediate restoration of the cartilage surface by treating the entire osteochondral unit. Nevertheless, it is a difficult, demanding technique, not without complications and requires joint harvesting. The limit of the technique is represented by the size of the lesion to be treated. The indication of choice is deep and small lesions (less than 4 $\mathrm{cm} 2)$ located on the medial condyle.

\section{REFERENCES:}

1. Magnussen R, Dunn W, Carey J, Spindler K. Treatment of focal articular cartilage defects in the knee. A systematic review. Clin Orthop Relat Res 2008;466:952-62.

2. Cole B, Pascual-Garrido C, Grumet R. Surgical management of articular cartilage defects in the knee. J Bone Joint Surg Am 2009;91:1778-90. 
3. Hangody L, Karpati Z. New possibilities in the management of severe circumscribed cartilage damage in the knee. Magy Traumatol Ortop Kezseb Plasztikai Seb 1994;37(3):237-43.

4. Vasiliadis HS, Wasiak J. Autologous chondrocyte implantation for full thickness articular cartilage defects of the knee.Cochrane Database Syst Rev 2010;6(10):CD003323.

5. Jakobsen B, Engebretsen L, Slauterbeck J. An analysis of the quality of cartilage repair studies. J Bone Joint Surg Am 2005;87(10):2232-8.

6. Hefti F, Beguiristain J, Krauspe R, et al. Osteochondritis dissecans:Amulticenter study of the European Pediatric Orthopedic Society. J Pediatr Orthop B 1999;8:23145.

7. Cepero S, Ullot R, Sastre S. Osteochondritis of the femoral condyles in the children and adolescents: our experience over the last 28 years. J Pediatr Orthop B 2005;14:24-9.

8. Lefort G, Moyen B. L'ostéochondrite disséquante des condyles fémoraux : analyse de 892 cas. Symposium de la Société franc, aise de chirurgie orthopédique. Rev Chir Orthop 2006;92:2S97-141.

9. Flynn JM, Kocher MS, Ganley TJ. Osteochondritis dissecans of the knee. J Pediatr Orthop 2004;24:434-41.

10. Yoshida S, Ikata T, Takai H, Kashiwaguchi S, Katoh S, Takeda Y. Osteochondritis dissecans of the femoral condyle in the growth stage. Clin Orthop 1998;346:162-70.

11. De Smett AA, Ilahi OA, Graf BK. Untreated osteochondritis dissecans of the femoral condyles: prediction of patient outcome using radiographic and MR findings. Skeletal Radiol 1997;26:463-7.

12. O'Connor MA, Palaniappan M, Khan N, Bruce CE. Osteochondritis dissecans of the knee in children. A comparison of MRI and arthroscopic findings. J Bone Joint Surg 2002;84B:258-62.

13. Pill SG, Ganley TJ, Milam RA, Lou JE, Meyer JS, Flynn JM. Role of magnetic resonance imaging and clinical criteria in predicting successful nonoperative treatment of osteochondritis dissecans in children. J Pediatr Orthop 2003;23:102-8.

14. Vasiliadis HS, Wasiak J. Autologous chondrocyte implantation for full thickness articular cartilage defects of the knee. Cochrane Database Syst Rev 2010;6(10):CD003323.

15. Garretson RB, Katolik LI, Verma N, Beck PR, Bach BR, Cole BJ. Contact pressure at osteochondral donor sites in the patellofemoral joint. Am J Sports Med 2004;32(4): 967-74. 
16. Reddy S, Pedowitz DI, Parekh SG, Sennett BJ, Okereke E. The morbidity associated with osteochondral harvest from asymptomatic knees for the treatment of osteochondral lesions of the talus. Am J Sports Med 2007;35(1):80-5.

17. Iwasaki N, Kato H, Kamishima T, Suenaga N, Minami A. Donor site evaluation after autologous osteochondral mosaicplasty for cartilaginous lesions of the elbow joint. Am J Sports Med 2007;35(12):2096-100.

18. Hangody L, Vásárhelyi G, Hangody LR, Sükösd Z, Tibay G, Bartha L, et al. Autologous osteochondral grafting - technique and long-term results. Injury 2008;39(Suppl. 1):S32-9.

19. Robert H. Technique de réparation du cartilage du genou par plastie en mosaïque. In: Conférence d'enseignement 2010 (99). Édition Elsevier Masson. Sous la direction de Denis Huten; Sofcot.

20. Sgaglione NA, Chen E, Bert JM, Amendola A, Bugbee WD. Current strategies for nonsurgical, arthroscopic, and minimally invasive surgical treatment of knee cartilage pathology. Instr Course Lect 2010;59:157-80.

21. Tetta C, Busacca M, Moio A, Rinaldi R, Delcogliano M, Kon E, et al. Knee osteochondral autologous transplantation: longterm MR findings and clinical correlations. Eur J Radiol 2010;76(1):117-23.

22. Gudas R, Kalesinskas RJ, Kimtys V, Stankevicius E, Toliusis V, Bernotavicius G, et al. A prospective randomized clinical study of mosaic osteochondral autologous transplant versus microfracture for the treatment of osteochondral defects in the knee joint in young athletes. Arthroscopy 2005;21: 1066-75.

23. Horas U, Pelinkovic D, Herr G, Aigner T, Schnettler R. Autologous chondrocyte implantation and osteochondral cylinder transplantation in cartilage repair of the knee joint. A prospective, comparative trial. J Bone Joint Surg Am 2003;85-A(2):185-92.

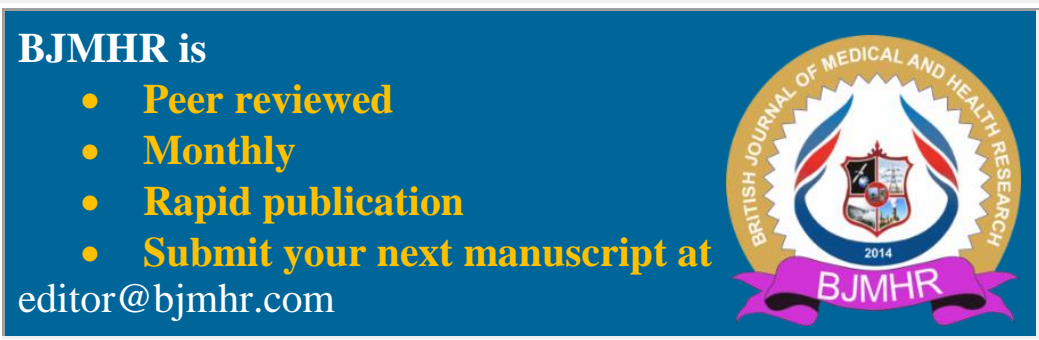

